\title{
Construction of Complete and Independent Systems of Rotation Moment Invariants
}

\author{
Jan Flusser and Tomáš Suk \\ Institute of Information Theory and Automation \\ Academy of Sciences of the Czech Republic \\ Pod vodárenskou věží 4, 18208 Prague 8, Czech Republic \\ $\{$ flusser, suk\}@utia.cas.cz \\ http://www.utia.cas.cz
}

\begin{abstract}
The problem of independence and completeness of rotation moment invariants is addressed in this paper. General method for constructing invariants of arbitrary orders by means of complex moments is described. It is shown that for any set of invariants there exists relatively small basis by means of which all other invariants can be generated. The method how to construct such a basis is presented. Moreover, it is proved that all moments involved can be recovered from this basis. The basis of the 3rd order moment invariants is constructed explicitly and its relationship to Hu's invariants is studied. Based on this study, Hu's invariants are shown to be dependent and incomplete.
\end{abstract}

\section{Introduction}

Moment invariants have become a classical tool for object recognition during last thirty years. They were firstly introduced to the pattern recognition community by $\mathrm{Hu}$ [1, who employed results of the theory of algebraic invariants and derived his seven famous invariants to translation, rotation, and scaling of $2-\mathrm{D}$ objects. Since that time, numerous papers have been devoted to various improvements and generalizations of the Hu's invariants but only few attempts to derive invariants from moments of orders higher than three have been done. $\mathrm{Li}$ [2] and Wong [3] presented the systems of invariants up to the orders nine and five, respectively. Unfortunately, no one of them paid attention to mutual dependence/independence of the invariants. The invariant sets presented in their papers are algebraically dependent. Most recently, Flusser 4] has proposed a method how to derive independent sets of invariants of any orders. However, in [4] the completeness was proven only within a certain class of invariants and the problem of recovering moments from the invariants was not investigated.

In this paper, we present a general method how to derive rotation moment invariants of any order. Furthermore, we show that there exists a relatively small set - basis - of the invariants which is independent and complete and we give an explicit algorithm for its construction. Knowing the invariants from the basis we can recover all moments involved and, consequently, we can express any existing moment invariant of any kind in terms of the basis elements. As 
a consequence, we show that most of the previously published sets of rotation moment invariants including Hu's system are dependent and/or incomplete. This is a surprising result giving a new look at Hu's invariants and possibly yielding a new interpretation of some previous experimental results.

\section{General Scheme for Deriving Invariants}

There are various approaches to the theoretical derivation of moment-based rotation invariants. $\mathrm{Hu}$ [1] employed the theory of algebraic invariants, $\mathrm{Li}$ [2] used the Fourier-Mellin transform, Teague [5] proposed to use Zernike moments, and Wong 3] used complex monomials which also originate from the theory of algebraic invariants. In this paper, we present a new scheme, which is based on complex moments. The idea to use the complex moments for deriving invariants was already described by Mostafa and Psaltis [6] but they concentrated themselves to the evaluation of the invariants rather than to constructing higher-order systems. In comparison with the previous approaches, this one is more transparent and allows to study mutual dependence/independence of the invariants in a readable way. It should be noted that all the above approaches differ from each other formally by mathematical tools and notation used but the general idea behind them is common and the results are similar or even equivalent.

Complex moment $c_{p q}$ of order $(p+q)$ of an integrable image function $f(x, y)$ is defined as

$$
c_{p q}=\int_{-\infty}^{\infty} \int_{-\infty}^{\infty}(x+i y)^{p}(x-i y)^{q} f(x, y) d x d y
$$

where $i$ denotes imaginary unit. Each complex moment can be expressed in terms of geometric moments $m_{p q}$ as

$$
c_{p q}=\sum_{k=0}^{p} \sum_{j=0}^{q}\left(\begin{array}{l}
p \\
k
\end{array}\right)\left(\begin{array}{l}
q \\
j
\end{array}\right)(-1)^{q-j} \cdot i^{p+q-k-j} \cdot m_{k+j, p+q-k-j},
$$

where geometric moments are defined as

$$
m_{p q}=\int_{-\infty}^{\infty} \int_{-\infty}^{\infty} x^{p} y^{q} f(x, y) d x d y
$$

To express geometric moments in terms of complex moments, we can substitute new variables for $(x+i y)$ and $(x-i y)$ into (1) and (3) and, consequently, derive an inverse form of eq. (2):

$$
m_{p q}=\frac{1}{2^{p+q} i^{q}} \sum_{k=0}^{p} \sum_{j=0}^{q}\left(\begin{array}{l}
p \\
k
\end{array}\right)\left(\begin{array}{l}
q \\
j
\end{array}\right)(-1)^{q-j} \cdot c_{k+j, p+q-k-j} .
$$

In polar coordinates, (1) becomes the form

$$
c_{p q}=\int_{0}^{\infty} \int_{0}^{2 \pi} r^{p+q+1} e^{i(p-q) \theta} f(r, \theta) d r d \theta .
$$


It follows from the definition that $c_{p q}=c_{q p}^{*}$ (the asterisk denotes complex conjugate). Furthermore, it follows immediately from (5) that the moment magnitude $\left|c_{p q}\right|$ is invariant to rotation of the image while the phase is shifted by $(p-q) \alpha$, where $\alpha$ is the angle of rotation. More precisely, it holds for the moment of the rotated image

$$
c_{p q}^{\prime}=e^{-i(p-q) \alpha} \cdot c_{p q} .
$$

Any approach to the construction of rotation invariants is based on a proper kind of phase cancellation. The simplest method proposed by many authors is to use the moment magnitudes themselves as the invariants. However, they do not generate a complete set of invariants. In the following Theorem, phase cancellation is achieved by multiplication of appropriate moment powers.

Theorem 1. Let $n \geq 1$ and let $k_{i}, p_{i}$, and $q_{i}(i=1, \cdots, n)$ be non-negative integers such that

$$
\sum_{i=1}^{n} k_{i}\left(p_{i}-q_{i}\right)=0
$$

Then any product

$$
I=\prod_{i=1}^{n} c_{p_{i} q_{i}}^{k_{i}}
$$

is invariant to rotation.

\section{Construction of the Basis}

Theorem 1 allows us to construct an infinite number of the invariants for any order of moments, but only few of them are mutually independent. By the term basis we intuitively understand the smallest set by means of which all other invariants can be expressed. More precisely, the basis must be independent, which means that none of its elements can be expressed as a function of the other elements, and also complete, which means that any rotation invariant can be expressed just by means of the basis elements.

The knowledge of the basis is a crucial point in all pattern recognition problems because it provides the same discriminative power as the set of all invariants at minimum computational costs. For instance, the set

$$
\left\{c_{20} c_{02}, c_{21}^{2} c_{02}, c_{12}^{2} c_{20}, c_{21} c_{12}, c_{21}^{3} c_{02} c_{12}\right\}
$$

is a dependent set whose basis is $\left\{c_{12}^{2} c_{20}, c_{21} c_{12},\right\}$.

Now we can formulate the fundamental theorem of this paper that tells us how to construct an invariant basis for a given set of moments.

Theorem 2. Let us consider complex moments up to the order $r \geq 2$. Let a set of rotation invariants $\mathcal{B}$ be constructed as follows:

$$
(\forall p, q \mid p \geq q \wedge p+q \leq r)\left(\Phi(p, q) \equiv c_{p q} c_{q_{0} p_{0}}^{p-q} \in \mathcal{B}\right),
$$


where $p_{0}$ and $q_{0}$ are arbitrary indices such that $p_{0}+q_{0} \leq r, p_{0}-q_{0}=1$ and $c_{p_{0} q_{0}} \neq 0$ for all images involved. Then $\mathcal{B}$ is a basis of a set of all rotation invariants created from the moments up to the order $r$.

Theorem 2 is very strong because it claims $\mathcal{B}$ is a basis of all possible rotation invariants, not only of those constructed according to (7). This is a significant difference from a similar theorem published in our recent paper [4].

Proof. The independence of $\mathcal{B}$ follows immediately from the mutual independence of the complex moments themselves (see 4 for details). To prove its completeness, it is sufficient to resolve so-called inverse problem, which means to recover all complex moments (and, consequently, all geometric moments) up to the order $r$ when knowing the elements of $\mathcal{B}$. Thus, the nonlinear system of equations

$$
\Phi(p, q)=c_{p q} c_{q_{0} p_{0}}^{p-q}
$$

must be resolved for each $c_{p q}$ involved.

Since $\mathcal{B}$ is a set of rotation invariants, it does not reflect the orientation of the object. Thus, there is one degree of freedom when recovering the object moments which corresponds to the choice of the object orientation. Without loss of generality, we can choose such orientation in which $c_{p_{0} q_{0}}$ is real and positive. As can be seen from eq. (6), if $c_{p_{0} q_{0}}$ is nonzero then such an orientation always exists. Thus, $c_{p_{0} q_{0}}$ can be calculated as

$$
c_{p_{0} q_{0}}=\sqrt{\Phi\left(p_{0}, q_{0}\right)}
$$

Consequently, using the relationship $c_{q_{0} p_{0}}=c_{p_{0} q_{0}}$, we get the solutions

$$
c_{p q}=\frac{\Phi(p, q)}{c_{q_{0} p_{0}}^{p-q}}
$$

and

$$
c_{p p}=\Phi(p, p)
$$

for any $p$ and $q$. Recovering the geometric moments is straightforward from eq. (4).

As an example, we present the basis of the invariants composed from the moments of 2 nd and 3rd orders, that is constructed according to Theorem 2 by choosing $p_{0}=2$ and $q_{0}=1$.

$$
\begin{aligned}
& \Phi(1,1)=c_{11}, \\
& \Phi(2,1)=c_{21} c_{12}, \\
& \Phi(2,0)=c_{20} c_{12}^{2}, \\
& \Phi(3,0)=c_{30} c_{12}^{3} .
\end{aligned}
$$




\section{Relationship to Hu's Invariants}

In this Section, we highlight the relationship between Hu's invariants 1] and the proposed invariants (9). We show Hu's invariants are incomplete and mutually dependent.

Let us recall the Hu's rotation invariants first:

$$
\begin{aligned}
\phi_{1}= & m_{20}+m_{02}, \\
\phi_{2}= & \left(m_{20}-m_{02}\right)^{2}+4 m_{11}^{2}, \\
\phi_{3}= & \left(m_{30}-3 m_{12}\right)^{2}+\left(3 m_{21}-m_{03}\right)^{2}, \\
\phi_{4}= & \left(m_{30}+m_{12}\right)^{2}+\left(m_{21}+m_{03}\right)^{2}, \\
\phi_{5}= & \left(m_{30}-3 m_{12}\right)\left(m_{30}+m_{12}\right)\left(\left(m_{30}+m_{12}\right)^{2}-3\left(m_{21}+m_{03}\right)^{2}\right) \\
& +\left(3 m_{21}-m_{03}\right)\left(m_{21}+m_{03}\right)\left(3\left(m_{30}+m_{12}\right)^{2}-\left(m_{21}+m_{03}\right)^{2}\right), \\
\phi_{6}= & \left(m_{20}-m_{02}\right)\left(\left(m_{30}+m_{12}\right)^{2}-\left(m_{21}+m_{03}\right)^{2}\right) \\
& +4 m_{11}\left(m_{30}+m_{12}\right)\left(m_{21}+m_{03}\right), \\
\phi_{7}= & \left(3 m_{21}-m_{03}\right)\left(m_{30}+m_{12}\right)\left(\left(m_{30}+m_{12}\right)^{2}-3\left(m_{21}+m_{03}\right)^{2}\right) \\
& -\left(m_{30}-3 m_{12}\right)\left(m_{21}+m_{03}\right)\left(3\left(m_{30}+m_{12}\right)^{2}-\left(m_{21}+m_{03}\right)^{2}\right) .
\end{aligned}
$$

It can be seen clearly that the Hu's invariants are nothing else than particular representatives of the general form (7):

$$
\begin{aligned}
\phi_{1} & =c_{11}, \\
\phi_{2} & =c_{20} c_{02}, \\
\phi_{3} & =c_{30} c_{03}, \\
\phi_{4} & =c_{21} c_{12}, \\
\phi_{5} & =\operatorname{Re}\left(c_{30} c_{12}^{3}\right), \\
\phi_{6} & =\operatorname{Re}\left(c_{20} c_{12}^{2}\right), \\
\phi_{7} & =\operatorname{Im}\left(c_{30} c_{12}^{3}\right) .
\end{aligned}
$$

Using (11) we can demonstrate the dependency of the Hu's invariants. It holds

$$
\phi_{3}=\frac{\phi_{5}^{2}+\phi_{7}^{2}}{\phi_{4}^{3}},
$$

which means that $\phi_{3}$ is useless and can be excluded from the Hu's system without any loss of discrimination power.

Moreover, the Hu's system is incomplete. Let us try to recover complex and geometric moments when knowing $\phi_{1}, \cdots, \phi_{7}$ under the same normalization constraint as in the previous case, i.e. $c_{21}$ is real and positive. Complex moments $c_{11}, c_{21}, c_{12}, c_{30}$, and $c_{03}$ can be recovered in a straightforward way but $c_{20}$ cannot be fully determined. Its real part is given unambiguously but the sign of its imaginary part (i.e. the sign of $m_{11}$ ) cannot be recovered. The incompleteness of the Hu's invariants implicates their lower discrimination power comparing to 


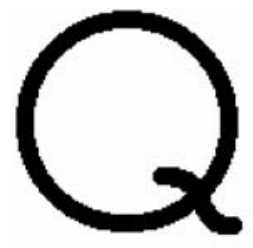

a

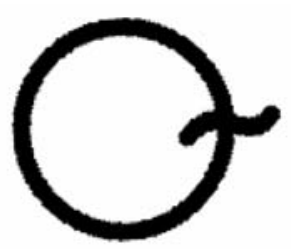

$\mathrm{b}$

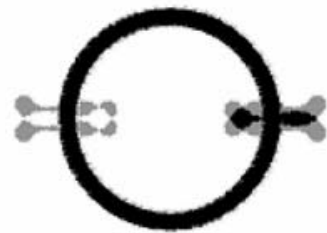

c

Fig. 1. The stylized letter Q: original (a), rotated to the normalized position (b), another object created according to (12) (c). For the values of the respective invariants see Table 1

Table 1. The values of the Hu's invariants and the new invariants (91) of the objects from Fig. 1 $\mathrm{b}$ and Fig. 15, respectively. The only invariant discriminating them is emphasized.

\begin{tabular}{|c|c|c||c|c|c|}
\hline $\mathrm{Hu}$ & Fig. 1b & Fig. 1 $1 \mathrm{~b}$ & New & Fig. 1b & Fig. 11 \\
\hline$\phi_{1}$ & 0.0032 & 0.0032 & $\Phi(1,1)$ & 0.0032 & 0.0032 \\
$\phi_{2}$ & $0.2128 \cdot 10^{-6}$ & $0.2128 \cdot 10^{-6}$ & $\Phi(2,1)$ & $0.7613 \cdot 10^{-9}$ & $0.7613 \cdot 10^{-9}$ \\
$\phi_{3}$ & $0.9717 \cdot 10^{-9}$ & $0.9717 \cdot 10^{-9}$ & $\operatorname{Re}(\Phi(2,0))$ & $0.3499 \cdot 10^{-12}$ & $0.3499 \cdot 10^{-12}$ \\
$\phi_{4}$ & $0.7613 \cdot 10^{-9}$ & $0.7613 \cdot 10^{-9}$ & $\operatorname{Im}(\mathbf{\Phi}(\mathbf{2}, \mathbf{0}))$ & $-\mathbf{0 . 2 9 9 3} \cdot \mathbf{1 0}^{-\mathbf{1 3}}$ & $\mathbf{0 . 2 9 5 4} \cdot \mathbf{1 0}^{-\mathbf{1 3}}$ \\
$\phi_{5}$ & $0.6609 \cdot 10^{-8}$ & $0.6609 \cdot 10^{-8}$ & $\operatorname{Re}(\Phi(3,0))$ & $0.6451 \cdot 10^{-18}$ & $0.6451 \cdot 10^{-18}$ \\
$\phi_{6}$ & $0.3499 \cdot 10^{-12}$ & $0.3499 \cdot 10^{-12}$ & $\operatorname{Im}(\Phi(3,0))$ & $-0.1123 \cdot 10^{-18}$ & $-0.1123 \cdot 10^{-18}$ \\
$\phi_{7}$ & $-0.1123 \cdot 10^{-18}$ & $-0.1123 \cdot 10^{-18}$ & & & \\
\hline
\end{tabular}

the proposed invariants of the same order. Let us consider two objects $a(x, y)$ and $b(x, y)$ in the normalized positions having the same geometric moments up to the third order except $m_{11}$, for which $m_{11}^{(a)}=-m_{11}^{(b)}$. In case of artificial data, such object $b(x, y)$ can be generated for any given $a(x, y)$ as

$$
b(x, y)=\frac{1}{2}(a(x, y)+a(-x, y)+a(x,-y)-a(-x,-y)),
$$

see Fig. 1 for an example. It is easy to prove that under (12) the moment constraints are always fulfilled. While the new invariants (9) distinguish these two objects by the imaginary part of the $\Phi(2,0)$, the Hu's invariants are not able to do so (see Table 1), even if the objects are easy to discriminate visually.

This property can be demonstrated also on real data. In Fig. 2 (left), one can see the photograph of a pan. A picture of a virtual "two-handle pan" (Fig. 2 right) was created from the original image according to (12). Although these two objects are apparently different, all their $\mathrm{Hu}$ 's invariants are exactly the same. On the other hand, the new invariant $\Phi(2,0)$ distinguishes these two objects clearly thanks to the opposite signs of its imaginary part. The same is true for the picture of a beater (see Fig. [3).

It should be noted that also other previously published systems of rotation invariants are dependent. Li [2] published a set of invariants from moments up to the 9th order. Unfortunately, his system includes the Hu's system as its subset 

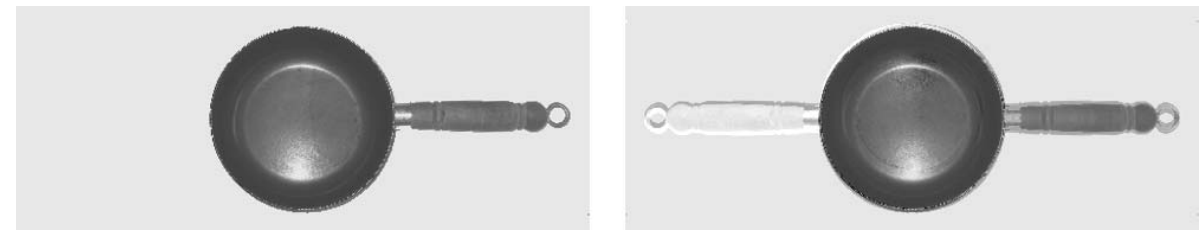

Fig. 2. Original image of a pan (left) and a virtual "two-handle pan" (right). These objects are distinguishable by the new invariants but not distinguishable by the Hu's invariants.
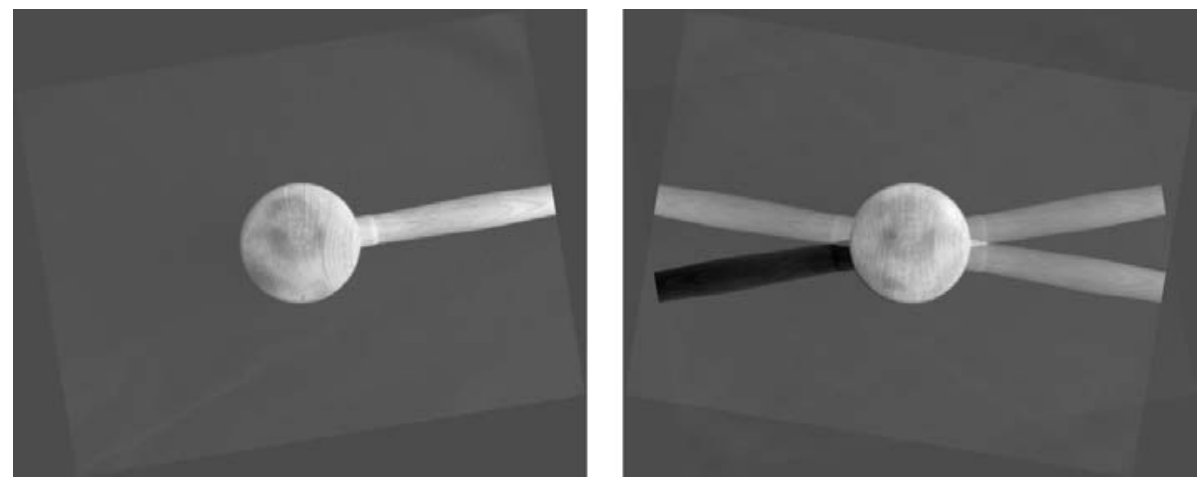

Fig. 3. Original image of a beater (left) and a virtual "four-handle beater" (right). These objects are distinguishable by the new invariants but not distinguishable by the Hu's invariants.

and therefore it also cannot be a basis. Wong [3] presented a set of 16 invariants from moments up to the 3rd order and a set of "more than 49" invariants from moments up to the 4th order. It follows immediately from Theorem 2 that a basis of the 3rd-order invariants has only 6 elements and a basis of the 4thorder invariants has 11 elements (these numbers relate to real-valued invariants). Thus, most of Wong's invariants are dependent and of no importance to practical pattern recognition problems.

\section{Conclusion}

In this paper, the problem of independence and completeness of the rotation moment invariants was discussed. Although the moment invariants have attracted significant attention of pattern recognition community within last thirty years, they have not been studied from this point of view yet.

The general method how to derive rotation invariants of any order was described first. Then the theorem presenting the smallest complete set (basis) of the invariants was formulated and proven. This is the major theoretical result of the paper. Finally, the relationship to the previous work was studied. It was shown that the proposed invariants outperform the widely-used Hu's moment invariants both in discrimination power and dimensionality requirements. 


\section{Acknowledgment}

This work has been supported by the grant No. 201/03/0675 of the Grant Agency of the Czech Republic.

\section{References}

1. M. K. Hu, "Visual pattern recognition by moment invariants," IRE Trans. Information Theory, vol. 8, pp. 179-187, 1962.

2. Y. Li, "Reforming the theory of invariant moments for pattern recognition," Pattern Recognition, vol. 25, pp. 723-730, 1992.

3. W. H. Wong, W. C. Siu, and K. M. Lam, "Generation of moment invariants and their uses for character recognition," Pattern Recognition Letters, vol. 16, pp. 115123, 1995.

4. J. Flusser, "On the independence of rotation moment invariants," Pattern Recognition, vol. 33, pp. 1405-1410, 2000.

5. M. R. Teague, "Image analysis via the general theory of moments," J. Optical Soc. of America, vol. 70, pp. 920-930, 1980.

6. Y. S. Abu-Mostafa and D. Psaltis, "Recognitive aspects of moment invariants," IEEE Trans. Pattern Analysis and Machine Intelligence, vol. 6, pp. 698-706, 1984. 